\title{
Topological Indices of Hyaluronic Acid-Paclitaxel Conjugates' Molecular Structure in Cancer Treatment
}

https://doi.org/10.1515/chem-2019-0009

received June 12, 2018; accepted September 3, 2018.

\begin{abstract}
A large number of medical experiments have confirmed that the features of drugs have a close correlation with their molecular structure. Drug properties can be obtained by studying the molecular structure of corresponding drugs. The calculation of the topological index of a drug structure enables scientists to have a better understanding of the physical chemistry and biological characteristics of drugs. In this paper, we focus on Hyaluronic Acid-Paclitaxel conjugates which are widely used in the manufacture of anticancer drugs. Several topological indices are determined by virtue of the edge-partition method, and our results remedy the lack of medicine experiments, thus providing a theoretical basis for pharmaceutical engineering.
\end{abstract}

Keywords: Topological Indices; Hyaluronic Acid; Paclitaxel; Hyaluronic Acid-Paclitaxel conjugates.

\section{Introduction}

With improvement in pharmaceutical technology in various countries in recent years a large proportion of newly emerging drugs are synthesized yearly from the laboratory and they are put on the market after clinical trials. At the initial stage a large number of experiments are required to test these new drugs to detect their biological activity, toxicity and degree of side effects on the human body. This results in a heavy workload in the laboratory, especially for countries and regions with low budgets and

\footnotetext{
*Corresponding author: Yiqiao Wang, School of Management, Beijing University of Chinese Medicine, Beijing 100029, China, E-mail:yqwang@bucm.edu.cn

Lina Zheng: College of Mathematics, Physics and Information Engineering, Zhejiang Normal University, Jinhua 321004, China Wei Gao: School of Information Science and Technology, Yunnan Normal University, Kunming 650500, China
}

poor experimental conditions (e.g., Southeast Asia, Africa, Latin America). In early chemical experiments, scientists compared a large amount of experimental data with the structure of compounds and concluded that the atomic arrangement of a compound had intrinsic relationships with the characteristics it exhibits (see Wiener [1] and Katritzky et al. [2] as examples). Based on this fact, as a branch of theoretical chemistry, through the analysis of the topological index on the molecular structure diagram of compounds and the corresponding properties of the compounds have been welcomed by more and more scientists and gradually applied to materials science, nanoscience, medicine, biology and pharmaceutical sciences (see Rehman et al. [3], Imran et al. [4], Baig et al. [5], Akhter et al. [6], Ali et al. [7], Kwun [8], Munir et al. [9], Riaz et al. [10] and Ma et al. [11]).

In computational modeling every atom is represented as a vertex and the covalent bond between atoms is expressed as an edge between two vertices. A graph obtained from a special chemical compound is called a molecular graph. Denote $G=(V(G), E(G))$ as a (molecular) graph, and here $V(G)$ and $E(G)$ are vertex set and edge set respectively, which is corresponding to atom set and chemical bond set.

Topological indices can be regarded as a large number of parameters on a molecular graph which is important in theoretical physics and pharmacology science. There are some important indices based on the vertex degree, e.g., Randić index, harmonic index, Zagreb indices, connectivity index (see Gao and Shi [12], and Gao and Wang [13, 14], and Gao et al. [15,16,17]), etc.

The famous Randić index [18], has been used effectively as a molecular descriptor in quantitative structurepharmacokinetics relationship (QSPR) and quantitative structure-activity relationship (QSAR) (see Pogliani [19], García-Domenech et al. [20], Stankevich et al. [21], Galvez [22], Estrada [23], Klein et al. [24]). If $G$ is a graph then its Randić index is denoted as: 


$$
R(G)=\sum_{u v E(G)}(d(u) d(v))^{-\frac{1}{2}}
$$

where $d(x)$ represents the degree of a vertex $x \hat{I} V(G)$.

In 1998, Bollobás and Erdös [25] generalized this index by replacing $-\frac{1}{2}$ with a real number $k$ as follows:

$$
R_{k}(G)=\sum_{u v E(G)}(d(u) d(v))^{k}
$$

The first three smallest general Randić indices of trees and corresponding extremal trees are determined by $\mathrm{Li}$ and Liu [26]. Liu and Gutman [27] estimated the general Randić index and some of its special cases, such as ordinary index and modified Zagreb index. When $k=1$ and $k=-1$, the second Zagreb index $M_{2}(G)$ and the modified second Zagreb index $M_{2}{ }^{*}(G)$ are written as:

$$
M_{2}(G)=\sum_{w \in E(G)} d(u) d(v),
$$

and

$$
M_{2}^{*}(G)=\sum_{w \in E(G)} \frac{1}{d(u) d(v)} .
$$

In 2009, Zhou and Trinajstić [28] defined the sumconnectivity index of a molecular graph $G$ as the following:

$$
\chi(G)=\sum_{u v E(G)}(d(u)+d(v))^{-\frac{1}{2}} .
$$

Replacing the value $-\frac{1}{2}$ by a real number $k$, we obtained the general sum connectivity index, which was expressed in [29]:

$$
\chi_{k}(G)=\sum_{u v E(G)}(d(u)+d(v))^{k}
$$

Moreover, Du, Zhou and Trinajstić [30] considered the smallest and the second smallest values of the general sum-connectivity indices of unicyclic graphs and studied the corresponding extremal graphs.

Given a molecular graph $G$, the harmonic index of $G$ is represented as:

$$
H(G)=\sum_{w \in E(G)} \frac{2}{d(u)+d(v)} .
$$

Favaron et al. [31] established the connections between the eigenvalues of molecular graphs and the harmonic index. Zhong [33] deduced the smallest and largest values of the harmonic index for connected molecular graphs and trees and characterized the corresponding extremal molecular graphs. Wu et al. [34] presented the minimum harmonic index of molecular graphs $\mathrm{G}$ with $\delta(G) \geq 2$. Liu [35] obtained some connections between the harmonic index and diameter of molecular graphs.

To expand the harmonic index to more chemical engineering applications, Yan et al. [32] investigated the general harmonic index:

$$
H_{k}(G)=\sum_{u \in E(G)}\left(\frac{2}{d(u)+d(v)}\right)^{k}
$$

Vuki cević and Furtula [36] proposed the geometricarithmetic index of a molecular graph $G$ as follows:

$$
G A(G)=\sum_{u v \in E(G)} \frac{2 \sqrt{d(u) d(v)}}{d(u)+d(v)}
$$

Eliasi and Iranmanesh [37] generalized the ordinary geometric-arithmetic index of a graph $G$ to the following form:

$$
O G A_{k}(G)=\sum_{u \in E(G)}\left(\frac{2 \sqrt{d(u) d(v)}}{d(u)+d(v)}\right)^{k}
$$

Azari and Iranmanesh [38] discussed the generalized Zagreb index as follows:

$$
M_{t_{1}, t_{2}}=\sum_{u \in E(G)}\left(d(u)^{t_{1}} d(v)^{t_{2}}+d(u)^{t_{2}} d(v)^{t_{1}}\right)
$$

where $t_{1}$ and $t_{2}$ are arbitrary non-negative integers.

Some polynomials connected on degree-based indices are also defined. For example, the first and the second Zagreb polynomials indices are as follows:

$$
M_{1}(G, x)=\sum_{u \in E(G)} x^{d(u)+d(v)}
$$

and

$$
M_{2}(G, x)=\sum_{u \in E(G)} x^{d(u) d(v)}
$$

Furthermore, the third Zagreb index and third Zagreb polynomial are defined as

$$
M_{3}(G)=\sum_{w \in E(G)}|d(u)-d(v)|
$$


and

$$
M_{3}(G, x)=\sum_{u \in E(G)} x^{|d(u)-d(v)|}
$$

respectively.

In the rest of the paper, we first introduce the polymers of Hyaluronic Acid-Paclitaxel conjugates and point out significance of this molecular structure. Then, in Section 3 , we present our main results and detailed proofs.

\section{Motivation}

Cancer is regarded as one of the major causes of death in the world and its death ratio continues to rise with the majority of deaths caused by breast, stomach, lung and colon cancers. Although there has been great developments in cancer biology and therapies to treat cancers, challenges still remain in primary and metastatic disease treatment. In addition, there are pitfalls in current anticancer drugs which induce low specificity and high toxicity thus severely limiting their efficacy. Certain advances have taken place in molecularly-targeted cancer treatment over recent years.

Hyaluronic acid (HA) is a compound that occurs naturally. It is a glycosaminoglycan polymer composed of a linear structure of units of D-glucuronic acid and N-acetyl-D-glucosamine, which are linked via alternating $\beta$-1,3- and $\beta$-1,4-glycosidic bonds. Its primary structure, disaccharide, is energetically stable [39]. $\mathrm{HA}$ is a promising cancer drug due to its unique, biodegradable, bio-compatible, nontoxic, hydrophilic, and non-immutable features; additionally, HA receptors demonstrated over-expression on many tumor cells. As a rapidly-growing platform for targeting CD44overexpressing cells nowadays, HA aims to improve anticancer therapies [40,41,42]. HA is an optimal drug carrier and drug targeting. Paclitaxel (PTX) is an effective drug, which is recommended for many types of cancers, including ovarian, breast, lung, bladder, prostate, and esophageal cancers, etc. [43]. While PTX administration also encounters its own limitations, such as its poor solubility and relevant side effects, as well as the excipients typically used in its formulation. Ringsdorf firstly proposed the method of developing polymeric macromolecule-drug conjugates, which was designed to deliver small hydrophobic drug molecules to their sites of action [44]. The main advantages of HA-PTX conjugates are the increase of its water solubility and the preservation of its activity, most importantly, it could be utilized as targeted drug delivery to reinforce anti-tumor efficacy [45,46,47]. The structure of hyaluronic acidpaclitaxel conjugates is depicted in Figure 1.

The study of these general indices for hyaluronic acid-paclitaxel conjugates meet certain limitations, even if some advances in degree-based indices of molecular graphs have been achieved in recent years. Moreover, as popular and critical drug structures, this polymer structure is widely applied in medical science and pharmaceutical fields. For instance, it is of great significance in the delivery system and in the development of anticancer drugs. These properties have attracted industrial interest and enormous academic interest to study the topological indices of this molecular structure from a mathematical perspective.

Ethical approval: The conducted research is not related to either human or animal use.

\section{Main Results and Proofs}

This section aims to present several generalized degreebased indices of hyaluronic acid-paclitaxel conjugates. The main technique used here is to classify edges into some parts. In the following discussion, let $G_{n}$ denote the molecular graph of HA-PTX conjugates with $n$ units' linear iteration. In figures 2 and 3, we depict their corresponding molecular graphs $G_{1}$ and $G_{3}$ when $n=1$ and $n=3$.

By observing the graph structure and calculating, we get $/ V\left(G_{n}\right) /=87 n$ and $/ E\left(G_{n}\right) /=96 n$.

If $\delta$ and $\Delta$ are the minimum and maximum degree of the graph $G_{n}$, respectively,we define:

(i) $E_{i}=\left\{u v \in E\left(G_{n}\right) / d(u)+d(v)=i\right\}$ for each $i$ with $2 \delta \leq i \leq 2 \Delta$;

(ii) $E_{j}^{*}=\left\{u v \in E\left(G_{n}\right) / d(u) d(v)=j\right\}$ for each $j, \delta^{2} \leq j \leq \Delta^{2}$.

Clearly, the sets of $E_{i}$ and $E_{j}^{*}$ may be non-empty.

Now that $\Delta=4$ and $\delta \geq 1$, we see that the edges of $G_{n}$ can be decomposed of the following subsets according to $E_{i}$ and $E_{j}^{*}$ definition:

$$
\begin{aligned}
& E_{3}=\left\{u v \in E\left(G_{n}\right) \mid d(u)=1, d(v)=2\right\} ; \\
& E_{4.1}=\left\{u v \in E\left(G_{n}\right) \mid d(u)=1, d(v)=3\right\} ; \\
& E_{4.2}=\left\{u v \in E\left(G_{n}\right) \mid d(u)=2, d(v)=2\right\} ; \\
& E_{5.1}=\left\{u v \in E\left(G_{n}\right) \mid d(u)=1, d(v)=4\right\} ; \\
& E_{5.2}=\left\{u v \in E\left(G_{n}\right) \mid d(u)=2, d(v)=3\right\} ; \\
& E_{6.1}=\left\{u v \in E\left(G_{n}\right) \mid d(u)=2, d(v)=4\right\} ; \\
& E_{6.2}=\left\{u v \in E\left(G_{n}\right) \mid d(u)=3, d(v)=3\right\} ; \\
& E_{7}=\left\{u v \in E\left(G_{n}\right) \mid d(u)=3, d(v)=4\right\} ; \\
& E_{8}=\left\{u v \in E\left(G_{n}\right) \mid d(u)=4, d(v)=4\right\} ; \\
& \text { Similarly, } \\
& E_{2}^{*}=\left\{u v \in E\left(G_{n}\right) \mid d(u)=1, d(v)=2\right\} ; \\
& E_{3}^{*}=\left\{u v \in E\left(G_{n}\right) \mid d(u)=1, d(v)=3\right\} ;
\end{aligned}
$$




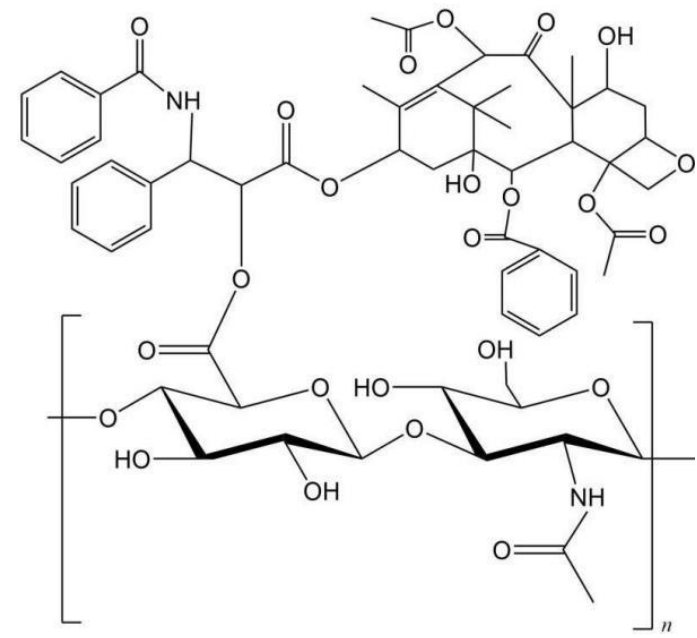

Figure 1: The molecular structure of hyaluronic acid-paclitaxel conjugates.

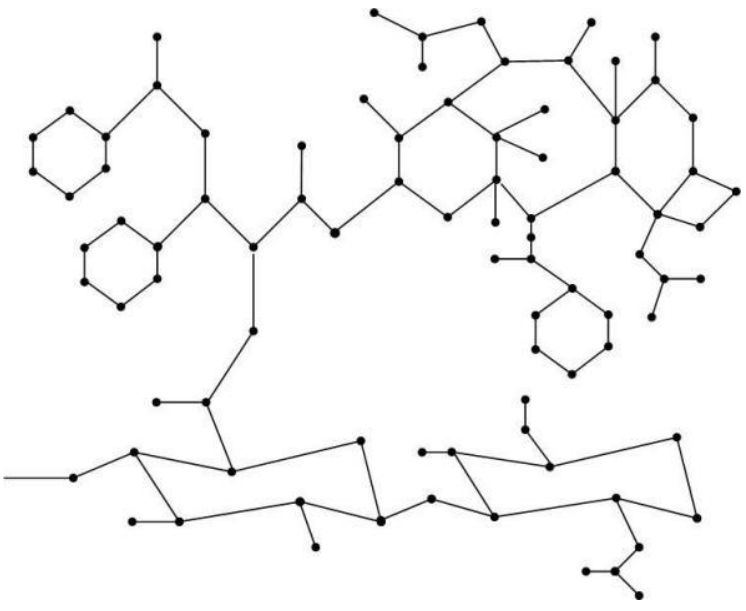

$E_{4.1}^{*}=\left\{u v \in E\left(G_{n}\right) \mid d(u)=1, d(v)=4\right\} ;$

$E_{4.2}^{*}=\left\{u v \in E\left(G_{n}\right) \mid d(u)=2, d(v)=2\right\}$;

$E_{6}^{*}=\left\{u v \in E\left(G_{n}\right) \mid d(u)=2, d(v)=3\right\}$;

$E_{8}{ }^{*}=\left\{u v \in E\left(G_{n}\right) \mid d(u)=2, d(v)=4\right\}$;

$E_{9}{ }^{*}=\left\{u v \in E\left(G_{n}\right) \mid d(u)=3, d(v)=3\right\}$;

$E_{12}{ }^{*}=\left\{u v \in E\left(G_{n}\right) \mid d(u)=3, d(v)=4\right\}$;

$E_{16}{ }^{*}=\left\{u v \in E\left(G_{n}\right) \mid d(u)=4, d(v)=4\right\}$.

Theorem 1 Let $G_{n}$ be the molecular graph of HA-PTX conjugates with $n$ units' linear iteration. Then, we have

$$
\begin{aligned}
R_{k}\left(G_{n}\right)= & 2 n \cdot 2^{k}+16 n \cdot 3^{k}+(17 n+1) \cdot 4^{k}+(33 n-1) \cdot 6^{k}+3 n \cdot 8^{k} \\
& +(19 n-1) \cdot 9^{k}+7 n \cdot 12^{k}+2 n \cdot 16^{k}
\end{aligned}
$$

Proof.

By graph structure analysis and observation, it is easy to deduce $\left|E_{3}\right|=\left|E_{2}{ }^{*}\right|=2 n,\left|E_{4.1}\right|=\left|E_{3}{ }^{*}\right|=16 n,\left|E_{5.1}\right|=\left|E_{4.1}{ }^{*}\right|=4 n$, $\left|E_{4.2}\right|=\left|E_{4.2}{ }^{*}\right|=13 n+1, \quad\left|E_{5.2}\right|=\left|\quad E_{6}{ }^{*}\right|=33 n-1, \quad\left|E_{6.1}\right|=\left|E_{8}^{*}\right|=3 n$, $\left|E_{6.2}\right|=\left|E_{9}{ }^{*}\right|=19 n-1,\left|E_{7}\right|=\left|E_{12}{ }^{*}\right|=7 n,\left|E_{8}\right|=\left|E_{16}{ }^{*}\right|=2 n$.

Thus, by the definition of general Randić and general sum connectivity index, we obtain

$$
\begin{aligned}
& R_{k}\left(G_{n}\right)=\sum_{u v \in E\left(G_{n}\right)}(d(u) d(v))^{k} \\
& =\sum_{u v \in E_{2^{*}}}(d(u) d(v))^{k}+\sum_{u v \in E_{3^{*}}}(d(u) d(v))^{k}+\sum_{u v \in E_{4.1}^{*}}(d(u) d(v))^{k} \\
& +\sum_{u v \in E_{42}^{*}}(d(u) d(v))^{k}+\sum_{u v \in E_{6^{*}}}(d(u) d(v))^{k}+\sum_{u v \in E_{8^{*}}}(d(u) d(v))^{k} \\
& +\sum_{u v \in E_{9^{*}}}(d(u) d(v))^{k}+\sum_{u v \in E_{1^{*}}}(d(u) d(v))^{k}+\sum_{u v \in E_{16^{*}}}(d(u) d(v))^{k} \\
& =2 n \cdot 2^{k}+16 n \cdot 3^{k}+(17 n+1) \cdot 4^{k}+(33 n-1) \cdot 6^{k}+3 n \cdot 8^{k}+(19 n-1) \cdot 9^{k} \\
& +7 n \cdot 12^{k}+2 n \cdot 16^{k}
\end{aligned}
$$

and

Figure 2: Corresponding molecular graph $\mathrm{G}_{1}: \mathrm{n}=1$.

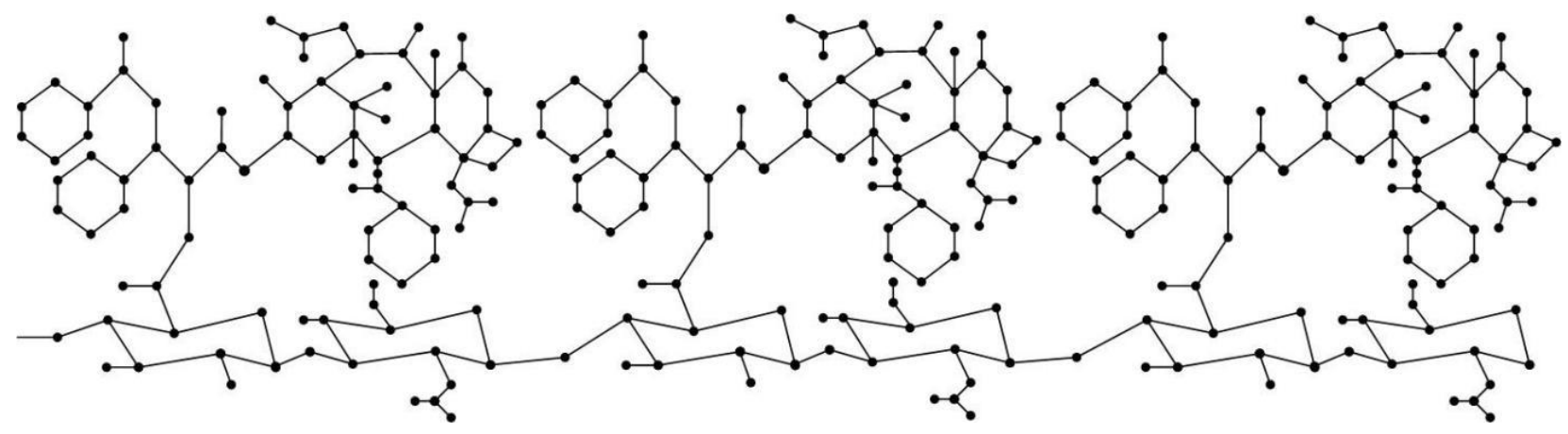

Figure 3: Corresponding molecular graph $G_{3}: n=3$. 
$\chi_{k}\left(G_{n}\right)=\sum_{u v \in E\left(G_{n}\right)}(d(u)+d(v))^{k}$

$=\sum_{u v \in E_{3}}(d(u)+d(v))^{k}+\sum_{u v \in E_{41}}(d(u)+d(v))^{k}+\sum_{u v \in E_{42}}(d(u)+d(v))^{k}$

$+\sum_{u v \in E_{51}}(d(u)+d(v))^{k}+\sum_{u v \in E_{5,2}}(d(u)+d(v))^{k}+\sum_{u v \in E_{61}}(d(u)+d(v))^{k}$

$+\sum_{u v \in E_{6,2}}(d(u)+d(v))^{k}+\sum_{u v \in E_{7}}(d(u)+d(v))^{k}+\sum_{u v \in E_{8}}(d(u)+d(v))^{k}$

$=2 n \cdot 3^{k}+(29 n+1) \cdot 4^{k}+(37 n-1) \cdot 5^{k}+(22 n-1) \cdot 6^{k}+7 n \cdot 7^{k}+2 n \cdot 8^{k}$

Hence, we get the desired conclusion.

Theorem 2 The general harmonic index of $G_{n}$ is

$$
\begin{aligned}
H_{k}\left(G_{n}\right) & =2 n \cdot\left(\frac{2}{3}\right)^{k}+(29 n+1) \cdot\left(\frac{1}{2}\right)^{k}+(37 n-1) \cdot\left(\frac{2}{5}\right)^{k}+ \\
& +(22 n-1) \cdot\left(\frac{1}{3}\right)^{k}+7 n \cdot\left(\frac{2}{7}\right)^{k}+2 n \cdot\left(\frac{1}{4}\right)^{k}
\end{aligned}
$$

Proof. By definition of general harmonic index and intermediate results manifested in Theorem 1 , we infer

$$
\begin{aligned}
& H_{k}\left(G_{n}\right)=\sum_{u v \in E\left(G_{n}\right)}\left(\frac{2}{d(u)+d(v)}\right)^{k} \\
& =\sum_{u v \in E_{3}}\left(\frac{2}{d(u)+d(v)}\right)^{k}+\sum_{u v \in E_{t 1}}\left(\frac{2}{d(u)+d(v)}\right)^{k}+\sum_{u v \in E_{42}}\left(\frac{2}{d(u)+d(v)}\right)^{k} \\
& +\sum_{u v \in E_{s 1}}\left(\frac{2}{d(u)+d(v)}\right)^{k}+\sum_{u v \in E_{s, 1}}\left(\frac{2}{d(u)+d(v)}\right)^{k}+\sum_{u v \in E_{61}}\left(\frac{2}{d(u)+d(v)}\right)^{k} \\
& +\sum_{u v \in E_{62}}\left(\frac{2}{d(u)+d(v)}\right)^{k}+\sum_{u v \in E_{7}}\left(\frac{2}{d(u)+d(v)}\right)^{k}+\sum_{u v \in E_{8}}\left(\frac{2}{d(u)+d(v)}\right)^{k} \\
& =2 n \cdot\left(\frac{2}{3}\right)^{k}+(29 n+1) \cdot\left(\frac{1}{2}\right)^{k}+(37 n-1) \cdot\left(\frac{2}{5}\right)^{k}+(22 n-1) \cdot\left(\frac{1}{3}\right)^{k}+7 n\left(\frac{2}{7}\right)^{k}+2 n\left(\frac{1}{4}\right)^{k} .
\end{aligned}
$$

We complete the proof.

By taking $k=1$, we yield the harmonic index of $G_{n}$.

\section{Corollary 3}

$$
H\left(G_{n}\right)=\frac{607}{15} n-\frac{7}{30} .
$$

In a similar fashion, we get the following conclusions on the exact expression of several important indices and polynomials of $G_{n}$.

Theorem 4 The ordinary geometric-arithmetic index of $G_{n}$ is

$$
\begin{gathered}
O G A_{k}\left(G_{n}\right)=5 n \cdot\left(\frac{2 \sqrt{2}}{3}\right)^{k}+16 n \cdot\left(\frac{\sqrt{3}}{2}\right)^{k}+4 n \cdot\left(\frac{4}{5}\right)^{k}+ \\
+(33 n-1) \cdot\left(\frac{2 \sqrt{6}}{5}\right)^{k}+7 n \cdot\left(\frac{4 \sqrt{3}}{7}\right)^{k}+34 n .
\end{gathered}
$$

Theorem 5 The generalized Zagreb index of $G_{n}$ is

$$
\begin{aligned}
M_{t_{1}, t_{2}}\left(G_{n}\right)= & 2 n \cdot\left(2^{t_{1}}+2^{t_{2}}\right)+16 n \cdot\left(3^{t_{1}}+3^{t_{2}}\right)+4 n \cdot\left(4^{t_{1}}+4^{t_{2}}\right)+(13 n+1) \cdot 2^{t_{1}+t_{2}+1} \\
& +(33 n-1) \cdot\left(2^{t_{1}} 3^{t_{2}}+2^{t_{2}} 3^{t_{1}}\right)+3 n \cdot\left(2^{t_{1}+2 t_{2}}+2^{t_{2}+2 t_{1}}\right)+2(19 n-1) \cdot 3^{t_{1}+t_{2}} \\
& +7 n \cdot\left(3^{t_{1}} 4^{t_{2}}+3^{t_{2}} 4^{t_{1}}\right)+4 n \cdot 4^{t_{1}+t_{2}} .
\end{aligned}
$$

Theorem 6 The first and second Zagreb polynomials of $G_{n}$ are

$$
\begin{gathered}
M_{1}\left(G_{n}, x\right)=2 n \cdot x^{3}+(29 n+1) \cdot x^{4}+(37 n-1) \cdot x^{5}+ \\
+(22 n-1) \cdot x^{6}+7 n \cdot x^{7}+2 n \cdot x^{8},
\end{gathered}
$$

and

$$
\begin{aligned}
M_{2}\left(G_{n}, x\right)= & 2 n \cdot x^{2}+16 n \cdot x^{3}+(17 n+1) \cdot x^{4}+(33 n-1) \cdot x^{6} \\
& +3 n \cdot x^{8}+(19 n-1) \cdot x^{9}+7 n \cdot x^{12}+2 n \cdot x^{16}
\end{aligned}
$$

Theorem 7 The third Zagreb index and polynomials of $G_{n}$ are

$$
M_{3}\left(G_{n}\right)=92 n+1
$$

and

$$
M_{3}\left(G_{n}, x\right)=(37 n-1) x+19 n x^{2}+4 n x^{3} .
$$

\section{Conclusion}

This work mainly aims to present the degree-based index of Hyaluronic Acid in light of molecular structural analysis, degree computation and mathematical derivation. The general Randić, general sum connectivity index, general harmonic index, ordinary geometricarithmetic index, generalized version of Zagreb index, first and second Zagreb polynomials, and the third Zagreb index and polynomial of HA-PTX $[n]$ are determined. The derived conclusions demonstrate the potential application prospects in pharmaceutical engineering in cancer treatment.

Conflict of Interests: We have no conflict of interests to declare.

Acknowledgements: We thank the reviewers for their constructive comments in improving the quality of this paper. This work was supported in part by the National Natural Science Foundation of China (11761083, 11771402, 11671053).

\section{References}

[1] Wiener H., Structural determination of paraffin boiling points, J. Amer. Chem. Soc., 1947, 69(1), 17-20.

[2] Katritzky A. R., Jain R., Lomaka A., Petrukhin R., Maran U., and Karelson M., Perspective on the relationship between melting 
points and chemical structure, Cryst. Growth Des., 2001, 1(4), 261-265.

[3] Rehman H. M. U., Sardar R., and Raza A., Computing topological indices of hex board and its line graph, Open J. Math. Sci., 2017, 1(1), 62-71.

[4] Imran M., Baig A. Q., Siddiqui H. M. A., and Sarwar R., On molecular topological properties of diamond-like networks, Can. J. Chem., 2017, 95(7), 758-770.

[5] Baig A. Q., Imran M., Khalid W., and Naeem N., Molecular description of carbon graphite and crystal cubic carbon structures, Can. J. Chem., 2017, 95(6), 674-686.

[6] Akhter S., Imran M., and Raza Z., Bounds for the general sumconnectivity index of composite graphs, J. Inequal. Appl., 2017, DOI: 10.1186/s13660-017-1350-y.

[7] Ali A., Nazeer W., Munir M., and Kang S. M., M-polynomials and topological indices Of zigzag and rhombic benzenoid systems, Open Chem., 2018, 16(1), 73-78.

[8] Kwun Y. C., Munir M., Nazcer W., Rafique S., and Kang S. M.. M-polynomials and topological indices of V-phenylenic nanotubes and nanotori, Scientific Reports, 2017, 7(1), DOI: 10.1038/s41598-017-08309-y.

[9] Munir M., Nazeer W., Kang S. M., Qureshi M. I., Nizami A. R., and Kwun Y. C., Some invariants of Jahangir graphs, Symmetry, 2017, 9(1), DOI: 10.3390/sym9010017.

[10] Riaz M., Gao W., and Baig A. Q., M-polynomials and degreebased topological indices of some families of convex polytopes, Open J. Math. Sci., 2018, 2(1), 18-28.

[11] Ma Y. D., Cao S. J., Shi Y. T., Gutman I., Dehmer M., and Furtula B., From the connectivity index to various Randic'-Type Descriptors, MATCH Commun. Math. Comput. Chem., 2018, 80(1), 85-106.

[12] Gao W. and Shi L., Wiener index of gear fan graph and gear wheel graph, Asian J. Chem., 2014, 26(11), 3397-3400.

[13] Gao W., Wang W. F., and Farahani M. R., Topological indices study of molecular structure in anticancer drugs, J. Chem., 2016, DOI:10.1155/2016/3216327.

[14] Gao W. and Wang W. F., The vertex version of weighted wiener number for bicyclic molecular structures, Comput. Math. Methods Med., 2015, DOI:10.1155/2015/418106.

[15] Gao W., Wang Y. Q., Wang W. F., and Shi L., The first multiplication atom-bond connectivity index of molecular structures in drugs, Saudi Pharm. J., 2017, 25(4), 548-555.

[16] Gao W. and Wang W. F., The fifth geometric-arithmetic index of bridge graph and carbon nanocones, J. Difference Equ. Appl., 2017, 23(1-2), 100-109.

[17] Gao W. and Wang W. F., The eccentric connectivity polynomial of two classes of nanotubes, Chaos Solitons Fractals, 2016, 89, 290-294.

[18] Randic'M., On characterization of molecular branching, J. Amer. Chem. Soc., 1975, 97, 6609-6615.

[19] Pogliani L., From molecular connectivity indices to semiempirical connectivity terms: Recent trends in graph theoretical descriptors, Chem. Rev., 2000, 100, 3287-3858.

[20] García-Domenech R., Galvez J., de Julián-Ortiz J. V., and Pogliani L., Some new trends in chemical graph theory, Chem. Rev., 2008, 108, 1127-1169.

[21] Stankevich V., Skvortsova M. I., and Zefirov N. S., On a quantum chemical interpretation of molecular connectivity indices for conjugated hydrocarbons, J. Mol. Struct., 1995, 342, 173-179.
[22] Galvez J., On a topological interpretation of electronic and vibrational molecular energies, J. Mol. Struct., 1998, 429, 255264.

[23] Estrada E., Physicochemical interpretation of molecular connectivity indices, J. Phys. Chem. A, 2002, 106(39), 90859091.

[24] Klein D. J., Palacios J. L., „Randic`M., and Trinajstić N., Random walks and chemical graph theory, J. Chem. Inf. Comp. Sci., 2004, 44, 1521-1525.

[25] Bollobás B. and Erdös P., Graphs of extremal weights, Ars Combin., 1998, 50, 225-233.

[26] Li B. J. and Liu W. J., The smallest Randić index for trees, Proc. Indian Acad. Sci. Math. Sci., 2013, 123(2), 167-175.

[27] Liu B. L. and Gutman I., Estimating the Zagreb and the general Randić indices, MATCH Commun. Math. Comput. Chem., 2007, 57(3), 617-632.

[28] Zhou B. and Trinajstić N., On a novel connectivity index, J. Math. Chem., 2009, 46(4), 1252-1270.

[29] Zhou B. and Trinajstić N., On general sum-connectivity index, J. Math. Chem., 2010, 47, 210-218.

[30] Du Z., Zhou B., and Trinajstić N., Minimum general sumconnectivity index of unicyclic graphs, J. Math. Chem., 2010, 48, 697-703.

[31] Favaron O., Maho M., and Sacle J. F., Some eigenvalue properties in graphs(conjectures of Graffiti-II), Discrete. Math., 1993, 111(13), 197-220.

[32] Yan L., Gao W., and Li J. S., General harmonic index and general sum connectivity index of polyomino chains and nanotubes, J. Comput. Theor Nanos, 2015, 12(10), 3940-3944.

[33] Zhong L., The harmonic index for graphs, Appl. Math. Lett., 2012, 25(3), 561-566.

[34] Wu R., Tang Z., and Deng H., A lower bound for the harmonic index of a graph with minimum degree at least two, Filomat, 2013, 27(1), 51-55.

[35] Lin J. X., On harmonic index and diameter of graphs, J. Appl. Math. Phys., 2013, 1(3), 5-6.

[36] Vukičević D. and Furtula B., Topological index based on the ratios of geometrical and arithematical means of end-vertex degrees of edges, J. Math. Chem., 2009, 46, 1369-1376.

[37] Eliasi M. and Iranmanesh A., On ordinary generalized geometricarithmetic index, Appl. Math. Lett., 2011, 24(4), 582-587.

[38] Azari M. and Iranmanesh A., Generalized Zagreb index of graphs, Studia Universitatis Babes-Bolyai Chemia, 2011, 56(3), 59-70.

[39] MráčekA., Varhaníková J., Lchocký M., Gřundělová L., Pokopcová A. and Velebný V., The influence of hofmeister series ions on hyaluronan swelling and viscosity, Molecules, 2008, 13, 10251034.

[40] Arpicco S., Milla P., Stella B., and Dosio F., Hyaluronic Acid Conjugates as a vectors for the active targeting of drugs, genes and nanocomposites in cancer treatment, Molecules, 2014,19, 3193-3230.

[41] Dosio F., Arpicco S., Stclla B., and Fattal E., Hyaluronic Acid for anticancer drug and nucleic acid delivery, Adv. Drug Deliver Rev., 2016, 97, 204-236.

[42] Zhang X. D., He F., Xiang K. Q., Zhang J. J., Xu M. Z., Long P. P., Su H.J., Gan Z.h., Yu Q.S., CD 44-targeted facile enzymatic activatable chitosan nanoparticles for efficient antitumor therapy and reversal of multidrug resistance, Biomacromolecules, 2018, 19, 883-895. 
[43] Mekhail T. M. and Markman M., Paclitaxel in cancer therapy, Expert Opin. Pharmacother., 2002, 3, 755-766.

[44] Ringsdorf H., Structure and properties of pharmacologically active polymers, J. Polymer Sci: Symposium, 1975, 51, 135-153.

[45] Lconelli F., Bella A. L., Migneco L. M., and Bettolo R. M., Design, synthesis and applications of hyaluronic acid-paclitaxel bioconjugates, Molecules, 2008, 13, 360-378.

[46] Xin D. C., Wang Y., and Xiang J. N., The use of amino acid linkers in the conjugation of paclitaxel with hyaluronic acid as drug delivery system: synthesis, self-assembled property, drug release, and in vitro efficiency, Pharm. Res., 2010, 27(2), 380389.

[47] Chen Y. J., Peng F. J., Song X. D., Wu J. C., Yao W. B., and Gao $X$. D., Conjugation of paclitaxel to C-6 hexanediamine-modified hyaluronic acid for targeted drug delivery to enhance antitumor efficacy, Carbohydr. Polym., 2018, 181, 150-158. 